\title{
S14. New perspectives in psychiatric epidemiology
}

\section{EPIDEMIOLOGY OF DEMENTIA AND ALZHEIMER'S DISEASE (AD) IN EUROPE}

L. Amaducci, M. Baldereschi

Department of Neurology, University of Florence, Viale Morgagni 85, 50134, Florence, Italy.

For most european countries there are just projections and estimations based on studies carried out in a few nations. In fact, Northern and Western Europe have made the major effort with a large number of population-based prevalence studies on dementia and Alzheimer's Disease. On the contrary, in Southem Europe epidemiological data are available only from Italy and Spain. Some of the european studies (11) were included in the EURODEM Concerted Action because of similar methodologies and comparability of the data. The meta-analysis of those data sets showed no major differences across the different countries. The prevalence rates by age turned out to be pretty similar. On the other hand, the relative prevalence of $A D$ and vascular dementia tumed out to be different across the EURODEM studies. It is generally believed that $\mathrm{AD}$ is the most common dementing disease, but in an Italian study vascular dementia was more frequent than $\mathrm{AD}$. The rate for AD could be lower than for other populations, or their rate for vascular dementia higher. As far as the data comparison cannot be fully reliable, the two possibilities cannot definitely be distinguished. There might be some environmental or genetic factors which account for such differences. A cross-national study using the same methodology in various countries is needed. Epidemiological studies using standard methodologies in different european community are all too rare. The only systematic cross-national study is the World Health Organization Age-Associated Dementia project that involves two european countries, Malta and Spain, as well as well as Chile, Nigeria and US. Many years were devoted to the development of the common methodology and epidemiological instruments as well as the cross-cultural standardization of the clinical diagnosis of dementia.

\begin{abstract}
CASUAL FACTORS IN THE EPIDEMIOLOGY OF MENTAL DISORDERS IN CHILDREN AND YOUTH

MH Schmidt

Central Institute of Mental Health, Department of Child Psychiatry, J5 D-68159 Mannheim (Germany)

Search for causes of psychiatric disorder in children or adolescents means searching for pathogenic conditions or mechanismus. They include today results of genetic epidemiology, mainly taken from adoption studies. Analytic epidemiology points out that acute life events at least in earlier developmental stages are less influential than chronic abnormal stress, this can be shown up clearly in parent's psychiatric disorder. Another aspect are the different effects of the socalled developmental tasks which may be experienced individually as either stressful or supportive. An appropriate paradigm for this are school influences. A third aspect are protective factors or mechanisms, which can be described as buffering stress. A closer look at this concept will demonstrate possibilities and its hazards.
\end{abstract}


NEURASTHENIA AND CHRONIC FATIGUE SYNDROME: The same but Different?

Simon Wessely

King's college Hospital and Institute of Psychiatry, Camberwel1, London SE5 9RS

This lecture will look at the links between the new condition of chronic fatigue syndrome (CFS), also known as post viral fatigue syndrome or ME, and the old condition of neurasthenia. A contrast will be drawn between what is known about operationally defined neurasthenia or CFS, and what is known about those who passionately believe they have CFS or ME. I will argue that although both are characterised by extreme physical and mental exhaustion arising after minimal effort, and moth are closely related to psychological disorders, the two conditions are also very different. These differences are to be found in the profound social and cultural divisions between the concepts, and in particular with the strong convictions of physical aetiology that go with the label of CFS/ME. Examples will be drawn from the media and popular literature to emphasise these differences. These distinctions are of interest to historians and sociologists, but also have profound effects on treatment and prognosis.

\section{EPIDEMIOLOGY OF SCHIZOPHRENIA}

H Häfner

Central Institute of Mental Health, J 5

D-68159 Mannheim, Federal Republic of Germany

Some recent results have aroused new hopes in the contribution of epidemiology to schizophrenia research: the transnational and crosscultural stability of the morbid risk and the steep decrease in first admission rates for schizophrenia since the middle of the sixties. Evidence of an increase in schizophrenic births $2-3$ months after the peak of the influenza epidemics in 1957 is another new challenge to schizophrenia research.

Clinical epidemiology furnished new insights in onset and early course. The first psychotic episode is preceded by a prodromal phase. The social sequelae of schizophrenia predominantly appear in the prodromal phase and thus prior to first hospitalization. Social disability and, under favourable environmental conditions, social status then remain stable in their mean values over very long time. The same is true for the positive and the negative symptomatology, part of the negative symptoms varying with the positive ones.

Current results of onset research show that at first onset women are four years older on average compared with man resulting in a later break in the social biography due to psychosis, consequently in less disruptions of the social development and in a more favourable social course. Beyond this indirect influence of age and sex, also genderrelated behaviour patterns and conditions of the social environment are effective which lead to quite different developments of various components of social status and thus also to differential gender differences in the course of schizophrenia.

\section{COMORBIDITY OF AFFECTIVE AND ANXIETY DISORDERS \\ J Angst \\ Psychiatric University Hospital Zurich, Research Department, P.O.Box 68, CH-8029 Zurich (Switzerland).}

Epidemiological research in the 1980s was dominated by large studies based on lay interviews carried out with the Diagnostic Interview Schedule (DIS) (Robins et al 1981) and the application of DSM-III diagnostic concepts and criteria. Lifetime prevalence rates of major depression were found to be within the range of 1 to $9 \%$. More recent studies, frequently carried out by professional interviewers, with the DIS or the Composite International Diagnostic Interview (CIDI) (WHO 1989) as well as with the Schedule of Affective Disorders or the SPIKE (Angst et al 1984), give consistently higher rates, mainly between 12 and $16 \%$ (exceptions go up to $25 \%$ ). Results of the ECA study (Weissman et al 1991) may therefore not be generalizable internationally. Lifetime prevalence rates of dysthymia are also still uncertain. They vary mainly between 1 and $9 \%$. Hypomania and bipolar disorder is the least well investigated affective group with prevalence rates between 0.1 and $7.5 \%$. There is good evidence for the assumption that hypomania is a heavily underestimated problem.

Among anxiety disorders a similar disparity of the finding is present, for instance panic disorders vary in their lifetime prevalence between $0.1 \%$ and $3.5 \%$, social phobia between 0.5 and $22.6 \%$. In face of these difficulties the strength of associations between affective disorders and anxiety disorders are frequently variable too and sample dependent.

Based on data from the prospective Zurich Study from age 20 to 30 longitudinal associations and especially follow-up data will be presented. In this context brief psychiatric syndromes (panic, brief hypomania, recurrent brief anxiety, recurrent brief depression and neurasthenia) can be distinguished from extended psychiatric syndromes (major depression, dysthymia, generalized anxiety and phobias). Longitudinal associations will be analyzed by follow-up data on diagnostic change and comorbidity. Comorbid conditions are more symptomatic and more severe in their consequences.

\section{EPIDEMIOLOGY OF SUICIDAL BEHAVIOUR IN EUROPE}

P Crepet $^{1}$ S Caracciolo ${ }^{2}$

IResearch Unit, EURO-WHO Multicentre Study on Parasuide, Emilia Romagna Regional Health Authority, via della Gensola 38, I-00153 Rome, Italy

2Department of Psychological Medicine, University of Ferrara, I-44100 Ferrara, Italy

Despite many methodological difficulties which make suicidal behaviour hard to be studied, there is no doubt that suicide is becoming one of the most dramatic social and sanitary emergencies. In fact, in the European Region every year about 135,000 people take their own lives and more than 900,000 attempt to do it. Suicidal behavour occurs mostly among the aged but, in the past decades, the most sharp increase appears to be found among the middle-aged and the young: as a result of this between 1970 and 1986 rates of suicide rose from 14.5 to 15.5 per 100,000 population which represents an overall $7 \%$ increase. From a geographic point of view, even if this increase is shared either by those already presenting high rates (Central Eastern and Nordic regions), or by those with low rates (Southern and Central Westem regions), the higher uprise is seen in the sub-regions where traditionally the rates used to be lower such as for instance Ireland and Italy where in the last three years the rates rose up to $20 \%$.

This paper does not only analyze suicide trends but also parasuicide. In the last four years parasuicide rates seem not to follow suicide rates. On the other hand the gap between sexes is closing up especially in the North of Europe. Some social and demographic characteristics are also presented in this paper.

The final part of this presentation will focus on prevention and the role of mental health services. In fact one data needs to be discussed: more than $60 \%$ of the people who have been admitted to a General Hospital emergency department for parasuicide have had either no previous psychiatric history nor have they after the attempt. This means that we should take into consideration a different way to plan and organize mental health facilities in order to offer a more effective answer to this particular population need. In this respect, some proposals are discussed. 